\title{
ORIGINAL
}

\section{PREVALENCIA DEL TABAQUISMO EN LOS TRABAJADORES DE UN HOSPITAL}

\section{José Miguel Arévalo Alonso y Francisco Javier Baquedano Arriazu}

Servicio de Medicina Preventiva y Salud Pública. Grupo de Prevención del uso del tabaco. Hospital de «Txagorritxu». Vitoria.

\section{RESUMEN}

Fundamento: Sobre tabaquismo se han realizado muchos estudios tanto en España como en otros países. El objetivo de esta encuesta es valorar la prevalencia de tabaquismo en una muestra representativa de trabajadores del hospital y su asociación con la edad, sexo. estamento profesional y actitudes.

Métodos: Después de un estudio piloto, se realizó una encuesta en una muestra representativa y aleatoria $(n=360)$ de trabajadores del hospital, a los que se les preguntó por sus actitudes al hábito tabáquico. clasificados por edad, sexo y estamentos.

Resultados: El porcentaje total de fumadores es de $36.4 \%$, pero no se han encontrado diferencias estadísticamente significativas ni por sexo ni grupo de edad. Los estamentos con mayor porcentaje de fumadores son ATS/DLE y Administrativos. El $42.8 \%$ de los no fumadores declaran baberlo hecho antes. siendo los estamentos de ATS/DUE, Administrativos y Facultativos los que mostraron el mayor porcentaje. Un $40.5 \%$ de fumadores declaran estar dispuestos a dejar el hábito, mientras el grupo de personas mayores de 50 años se muestran más resistentes. La mayoría de fumadores de cigarrillos señalan que fuman en el hospital. La mayor parte de ello; son fumadores intermedios (10-20 cigarrillos/día) y lo llevan haciendo muchos años. La mayor parte de los exfumadores declaran haber dejado el hábito en los últimos años.

Conclusiones: Estos resultados demuestran que un gran porcentaje de trabajadores del hospital son fumadores. Sin embargo. existe un importante número que está dispuestos a dejarlo. Por ello, animamos a estas personas a que dejen de fumar y ayudarles por medio de diversas acciones tales como: consulta de tratamiento del tabaquismo, consejo médico y educación para la salud. Proponemos declarar al hospital como «OSPITAL SIN TABACO para el año 2000, lo cual incluye a trabajadores, pacientes y visitas, de acuerdo con la estrategia de la OMS «Salud para todos».

Palabras clave: Prevalencia. Tabaquismo. Encuesta. Trabajadores del hospital.

\section{ABSTRACT \\ Prevalence of Tobaccoism amongst Hospital Workers}

Background: In Spain, as in other countries, numerous studies on tobaccoism have been carried out. The purpose of this survey was to evaluate the prevalence of tobaccoism in a representative sample of hospital employees and its association with age, sex, profession level and attitudes.

Methods: Following a pilot study, a survey was carried out amongst a representative and random sample $(n=360)$ of hospital personnel, who were asked about their attitudes towards smoking, classified according to age, sex and professional level.

Results: The total percentage of smokers was $36.4 \%$, but no significant statistical differences were found between sex or age group. The professional levels with the highest percentage of smokers are ATS/DUE and clerks. $42.8 \%$ of non-smokers declared having smoked in the past. ATS/DUE, clerks and doctors showed the highest percentage. $40.5 \%$ of smokers declare that they were willing to give up smoking, whereas people over 50 were the most reluctant. The majority of cigarette smokers admitted that they smoke in the hospital. Most of them are intermediate smokers (10-20 cigarettes per day) and have been so for many years. The majority of former smokers gave up smoking in recent years.

Conclusions: These results prove that a large number of hospital employees are smokers. However, many are willing to give up the habit. We should therefore encourage these people to give up smoking and help them by different means such as: anti-smoking advice, medical advice and health education. We propose 10 declare hospitals as «Non-Smoking Area» as of the year 2000 which includes employees, patients and visitors, according to the WHO «Health for All.» program.

Key words. Prevalence. Tobaccoism. Survey. Hospital workers.

Correspondencia:

JM Arévalo

Plaza Santa Bárbara, 8-4..$^{\circ}$ cha

01004 Vitoria

Fax: (945) 249473 


\section{INTRODUCCIÓN}

Las investigaciones epidemiológicas sobre el hábito tabáquico se iniciaron en 1950 con el clásico estudio de Doll y Hill que analizaba la asociación entre consumo de tabaco y carcinoma de pulmón en médicos del Reino Unido'. Estos mismos autores continuaron sus investigaciones estableciendo la relación entre tabaquismo, mortalidad por cáncer de pulmón ${ }^{2}$ y por otras causas ${ }^{3}$. Posteriormente se han venido realizando numerosos estudios sobre este hábito por diversos grupos en muchos países del mundo, tanto en población general como en grupos específicos tales como adolescentes, estudiantes, trabajadores y personal sanitario.

Todo ello nos ha permitido conocer la prevalencia del hábito tabáquico en los diversos colectivos y la descripción de las variables asociadas (edades, sexo, ocupación), así como la evolución del tabaquismo, comparación con otros países y/o colectivos, relación entre hábitos y patología (cáncer, cardiopatía isquémica, broncopatías, úlcera gastroduodenal), así como la influencia de los programas de educación sanitaria en la cesación del hábito tabáquico.

Estudios sobre el consumo de cigarrillos y otros hábitos se han relacionado con el estado de salud en el Proyecto Alameda County ${ }^{4}$. Se ha demostrado la relación del tabaco con una serie de enfermedades ${ }^{5}$ entre ellas las patologías cardiovasculares, como ha puesto de manifiesto el estudio Framinghan ${ }^{6}$.

El objetivo de nuestro estudio es valorar la prevalencia del hábito tabáquico en una muestra representativa de trabajadores de hospital y su asociación con la edad, categoría profesional, sexo y actitud sobre el consumo de tabaco en el centro.

\section{PERSONAS Y MÉTODOS}

A finales de 1993 se creó en el hospital el grupo de prevención del hábito tabáquico, formado por trabajadores de distintos esta- mentos, sensibilizados con este problema, con objeto de servir de base para posibles acciones preventivas enfocadas a disminuir el tabaquismo en el centro. En el seno de este grupo, el servicio de medicina preventiva y salud pública, propuso como una de las acciones a desarrollar, la realización de una encuesta para valorar la situación del tabaquismo entre los trabajadores del hospital.

A tal efecto, se confeccionó un cuestionario con las siguientes características: anónimo, fácil de rellenar, que su cumplimentación llevara poco tiempo y que abarcara a todos los estamentos profesionales del hospital. El estudio se llevó a efecto entre los años 1994 y 1995 en dos fases:

Estudio piloto: su finalidad fue doble: valorar la factibilidad de la encuesta y, basándose en los datos obtenidos en un pequeño número de trabajadores, realizar una predeterminación del tamaño de la muestra con objeto de que hubiera un número de personas suficientes para permitir la extrapolación de los resultados a todo el colectivo.

Para llevar a efecto este estudio, se solicitó a la dirección de personal la distribución de los trabajadores del centro por estamento profesional y sexo (tabla 1). Para formar parte de la encuesta piloto se seleccionó por muestreo aleatorio, estratificado, sistemático y simple al $10 \%$ de los trabajadores (teniendo en cuenta el estamento y sexo). Estas personas fueron invitadas, por parte de los miembros del grupo de trabajo de prevención del tabaco, a contestar la encuesta. El número total de encuestados fue de 110 (70 mujeres y 40 hombres).

Estudio principal: de acuerdo con el estudio piloto se procedió, en primer lugar, a modificar algunos aspectos del cuestionario, con lo que se estructuró el definitivo. Con objeto de disponer de una muestra representativa a partir de los resultados obtenidos en el estudio piloto y teniendo en cuenta el error muestral para muestras finitas con un nivel de confianza del $95 \%$, ajustando el número en relación con la composición proporcional 
Tabla 1

Distribución de trabajadores por estamento y sexo

\begin{tabular}{|c|c|c|c|c|c|c|}
\hline & \multicolumn{2}{|c|}{ TOTAL } & \multicolumn{2}{|c|}{ MUJERES } & \multicolumn{2}{|c|}{ HOMBRES } \\
\hline & $N^{o}$ & Pred. & $N^{o}$ & Pred. & $N^{o}$ & Pred. \\
\hline Directivos & 7 & - & 3 & - & 4 & - \\
\hline Facultativos & 228 & 62 & 71 & 14 & 157 & 48 \\
\hline Residentes & 78 & 19 & 45 & 9 & 33 & 10 \\
\hline ATS/DUE & 505 & 105 & 489 & 100 & 16 & 5 \\
\hline Aux. Enfermería & 347 & 68 & 346 & 68 & 1 & - \\
\hline Celadores & 113 & 30 & 11 & 2 & 102 & 28 \\
\hline Cocina/Lavandería & 135 & 27 & 128 & 25 & 7 & 2 \\
\hline Mantenimiento & 53 & 16 & - & $\ldots$ & 53 & 16 \\
\hline Administrativos & 154 & 33 & 127 & 25 & 27 & 8 \\
\hline TOTALES & 1620 & 360 & 1220 & 243 & 400 & 117 \\
\hline
\end{tabular}

N. ${ }^{\circ}$ : trabajadores del centro

Pred.: n. $^{\circ}$ de trabajadores predeterminados para formar parte de la muestra

por estamentos y sexo, se realizó la predeterminación del tamaño de la muestra necesaria $^{7}$, que resultó ser de 360 personas. La distribución por estamento y sexo se resume en la tabla 1.

Para seleccionar aleatoriamente a las personas integrantes de esta muestra representativa, se ha utilizado una tabla informatizada de números aleatorios por estamento y sexo ${ }^{8}$. El cuestionario utilizado se recoge en el anexo 1.

\section{Valoración estadística}

Se realizó un análisis bivariante de asociación entre las diversas conductas (fumar actualmente, haber furnado antes, estar dispuestos a dejar el tabaco) y las variables sociodemográficas (sexo y edad agrupadas cn 4 categorías, y estamentos profesionales clasificados en 8). Se calculó para cada variable la Odds Ratio (OR) con su correspondiente intervalo de confianza al $95 \%$

Anexo 1

Encuesta sobre hábito de fumar

edad

sexo

estamento

si fima actualmente:

cantidad / día

ocasional

tipo

tiempo

ifuma en el hospital?

¿está Vd. dispuesto/a a dejar de fumar")

¿por motivo del tabaco ha tenido algún problema médico?

¿en qué lugar?

¿cuál?

Si no fuma actualmente:

¿ha fumado antes?

cantidad / día

ocasional

tipo

tiempo

¿cuántos años lleva sin fumar? 
(IC95\%). Las variables con más de 2 categorías, se ordenaron de menor a mayor y se calculó la OR de tendencia lineal para todas ellas. La significación estadística se evaluó por medio de la prueba Chi-cuadrado, rechazándose la hipótesis nula para una $\mathrm{p}<0.05$. Para identificar las variables y sus categorías asociadas a las diversas conductas ajustadas por todas las demás, se han utilizado modelos de regresión logística múltiple.

Se utilizó el programa EPI-INFO versión 5 (CDC) para el análisis bivariante y el Módulo Logit del programa SYSTAT ver 5.03 (1991) para cálculo de modelos de regresión logística múltiple.

\section{RESULTADOS}

De los trabajadores encuestados declaran fumar el $36.4 \%$. No se evidencian diferencias estadísticamente significativas entre fumadores de ambos sexos (tabla 2). En cuanto a los grupos de edad el porcentaje de fumadores es mayor entre 31 a 40 años y menor en edades superiores a 50 años, aunque las diferencias entre sus OR de tendencia lineal no resultan estadísticamente significativas. Por estamentos ordenados de menor a mayor, según el porcentaje de fumadores y tomando como referencia el grupo de menor porcentaje (cocina/lavandería), pueden observarse diferencias estadísticamente significativas de tendencia lineal en los dos estamentos de más elevada proporción de fumadores (ATS/DUE y administrativos).

Tabla 2

Variables y categorías asociadas al hábito de fumar en el momento de la encuesta

\begin{tabular}{|c|c|c|c|c|c|c|c|}
\hline & \multicolumn{2}{|c|}{ Total encuestados } & \multicolumn{2}{|c|}{ Fuman actualmente } & \multirow{2}{*}{$O R$} & \multirow{2}{*}{ IC95\% } & \multirow{2}{*}{$p$} \\
\hline & $n$ & $\%(*)$ & $n$ & $\%(* *)$ & & & \\
\hline Total encuestados & 360 & - & 1,31 & 36.4 & & & \\
\hline \multicolumn{8}{|l|}{ SEXO } \\
\hline Masculino & 117 & 32.5 & $4 ?$ & 36.8 & & & \\
\hline Femenino & 243 & 67.5 & 88 & 36.2 & 1.0 & $0.6-1.7$ & .921 \\
\hline \multicolumn{8}{|l|}{ GRUPO ETARIO } \\
\hline$<31$ años & 39 & 10.8 & 13 & 33.3 & 1 & & \\
\hline $31-40$ años & 153 & 42.5 & 65 & 42.5 & 1.5 & $0.7-3.1$ & .301 \\
\hline 41-50 años & 111 & 30.8 & 39 & 35.1 & 1.1 & $0.5-2.3$ & .839 \\
\hline$>50$ años & 57 & 15.8 & 14 & 24.6 & 0.7 & $0.3-1.6$ & .349 \\
\hline \multicolumn{8}{|l|}{ ESTAMENTO } \\
\hline Cocina/lavandería & 27 & 7.5 & 5 & 18.5 & 1 & & \\
\hline Residentes & 19 & 5.3 & 4 & 21.1 & 1.2 & $0.3-5.1$ & .831 \\
\hline Aux. Enfermería & 68 & 18.9 & 17 & 25.0 & 1.5 & $0.5-4.5$ & .501 \\
\hline Mantenimiento & 16 & 4.4 & 4 & 25.0 & 1.5 & $0.3-6.5$ & .615 \\
\hline Celadores & 30 & 8.3 & 10 & 33.3 & 2.2 & $0.6-7.5$ & .210 \\
\hline Facultativos & 62 & 17.2 & 22 & 35.5 & 2.4 & $0.8-7.3$ & .116 \\
\hline ATS/DUE & 105 & 29.2 & 50 & 47.6 & 4.0 & $1.4-11.4$ & .009 \\
\hline Administrativos & 33 & 9.2 & 19 & 57.6 & 6.0 & $1.8-19.7$ & .003 \\
\hline \multicolumn{8}{|c|}{ ANÁLISIS DE REGRESIÓN LOGÍSTICA MÚLTIPLE } \\
\hline ESTAMENTO & \multicolumn{2}{|c|}{$O R$} & \multicolumn{2}{|c|}{ IC $95 \%$} & \multicolumn{3}{|c|}{$p$} \\
\hline Cocina/lavandería & \multicolumn{2}{|c|}{1} & \multirow{2}{*}{\multicolumn{2}{|c|}{$1.3-4.0$}} & \\
\hline ATS/DUE & \multicolumn{2}{|c|}{2.3} & & & \multicolumn{3}{|c|}{.003} \\
\hline Administrativos & \multicolumn{2}{|c|}{3.1} & \multicolumn{2}{|c|}{$1.5-6.2$} & & .002 & \\
\hline
\end{tabular}

(*) \% de cada categoría (dentro de cada varable).

(**) \% de los que fuman de cada categoria. 
Al valorar la asociación entre el hábito de fumar por cada categoría ajustada a todas las demás por medio del análisis de regresión logística múltiple, y utilizando como referencia en el caso de los grupos de edad los menores de 31 años y en el de estamentos profesionales el de menor porcentaje, se confirma la diferencia estadísticamente significativa de los grupos de ATS/DUE y per- sonal administrativo, si bien la OR es menor en ambos casos, que en el análisis bivariante. En la tabla 2 y para la regresión logística múltiple sólo se indican aquellas categorías que resultaron significativas en el análisis bivariante.

En la tabla 3 se resumen los datos correspondientes a los trabajadores que no fu-

Tabla 3

Variables y categorías asociadas en los exfumadores

\begin{tabular}{|c|c|c|c|c|c|c|c|}
\hline & \multicolumn{2}{|c|}{ No fumadores actuales } & \multicolumn{2}{|c|}{ Han fumado anteriormente } & \multirow{2}{*}{$O R$} & \multirow{2}{*}{$I C 95 \%$} & \multirow{2}{*}{$p$} \\
\hline & $n$ & $\%(*)$ & $n$ & $\%(* *)$ & & & \\
\hline Total & 229 & 63.6 & 98 & 42.8 & & & \\
\hline \multicolumn{8}{|l|}{ SEXO } \\
\hline Masculino & 74 & 63.2 & 39 & 52.7 & & & \\
\hline Femenino & 155 & 63.8 & 59 & 38.1 & 1.8 & $1.0-3.3$ & .037 \\
\hline \multicolumn{8}{|l|}{ GRUPO ETARIO } \\
\hline$<31$ años & 26 & 66.7 & 6 & 23.1 & 1.0 & & \\
\hline $31-40$ años & 88 & 57.5 & 43 & 48.9 & 3.2 & $1.2-8.7$ & .024 \\
\hline $41-50$ años & 72 & 64.9 & 33 & 45.8 & 2.8 & $1.0-7.8$ & .047 \\
\hline$>50$ años & 43 & 75.4 & 16 & 37.2 & 2.0 & $0.7-5.9$ & .226 \\
\hline \multicolumn{8}{|l|}{ ESTAMENTO } \\
\hline Cocina/lavandería & 22 & 81.5 & 3 & 13.6 & 1.0 & & \\
\hline Residentes & 15 & 79.0 & 4 & 26.7 & 2.3 & $0.4-12.2$ & .328 \\
\hline Aux. Enfermería & 51 & 75.0 & 14 & 27.5 & 2.4 & $0.6-9.4$ & .209 \\
\hline Celadorcs & 20 & 66.7 & 7 & 35.0 & 3.4 & $0.7-15.7$ & .115 \\
\hline Mantenimiento & 12 & 75.0 & 5 & 41.7 & 4.5 & $0.9-24.1$ & .077 \\
\hline ATS/DUE & 55 & 52.4 & 30 & 54.5 & 7.6 & $2.0-28.7$ & .003 \\
\hline Administrativos & 14 & 42.4 & 9 & 64.3 & 11.4 & $2.2-58.5$ & .004 \\
\hline Facultativos & 40 & 64.5 & 26 & 65.0 & 11.8 & $3.0-46.7$ & .000 \\
\hline \multicolumn{8}{|c|}{ ANÁLISIS DE REGRESIÓN LOGÍSTICA MÚLTIPLE } \\
\hline & \multicolumn{2}{|c|}{ OR } & \multicolumn{2}{|c|}{ IC $95 \%$} & \multicolumn{3}{|c|}{$p$} \\
\hline \multicolumn{8}{|l|}{ SEXO } \\
\hline Femenino & \multicolumn{2}{|c|}{1} & & & & & \\
\hline Masculino & & & \multicolumn{2}{|c|}{$0.6-4.7$} & \multicolumn{3}{|c|}{.367} \\
\hline \multicolumn{8}{|l|}{ EDAD } \\
\hline$<31$ & \multicolumn{2}{|c|}{1} & & & & & \\
\hline $31-40$ & \multirow{2}{*}{\multicolumn{2}{|c|}{$\begin{array}{l}2.2 \\
2.3\end{array}$}} & \multicolumn{2}{|c|}{$0.8-6.4$} & \multicolumn{3}{|c|}{.136} \\
\hline $41-50$ & & & \multicolumn{2}{|c|}{$0.8-6.6$} & \multicolumn{3}{|c|}{.136} \\
\hline \multicolumn{8}{|l|}{ ESTAMENTO } \\
\hline Cocina/lavandería & \multicolumn{2}{|c|}{1} & & & & & \\
\hline ATS/DUE & \multicolumn{2}{|c|}{2.3} & \multicolumn{2}{|c|}{$1.2-4.7$} & \multicolumn{3}{|c|}{.019} \\
\hline Administrativos & & & & & & .026 & \\
\hline Facultativos & & & & & & .029 & \\
\hline
\end{tabular}

(*) \$ sobre iotal de encuestados por categorici.

(**) \% sobre no fumadores actuales por categoria. 
mando en la actualidad, declaran haber fumado antes. El porcentaje de exfumadores es significativamente mayor en el sexo masculino. Por grupos de edad y tomando como referencia el de menor edad, se evidencia una $O R$ de tendencia lineal superior en el resto de grupos, resultando estadísticamente significativa en el comprendido entre 31 y 40 años y en cl límitc sc sitúan los pertenecientes a la década siguiente. Por estamentos y tomando como referencia el de menor porcentaje de los que declaran haber fumado antes (cocina/lavandería), se observan diferencias estadísticamente significativas en los colectivos de
ATS/DUE, administrativos y sobre todo en el de facultativos.

$\mathrm{Al}$ valorar la asociación por categorías entre los que han fumado anteriormente y no lo han hecho, ajustadas a todas las demás, tomando como referencia la de menor porcentaje, puede verse como desaparecen las diferencias por sexo y grupos de edad que fucron significativas en el análisis bivariante y se conservan en los tres estamentos, pero con un nivel de significación estadística menor.

La tabla 4 presenta la distribución de aquellos fumadores que declaran estar dispuestos a

Tabla 4

Variables y categorías asociadas a los que fumando actualmente declaran estar dispuestos a dejar el hábito

\begin{tabular}{|c|c|c|c|c|c|c|}
\hline & \multirow{2}{*}{ Total fumadores } & \multicolumn{2}{|c|}{ Fumadores dispuestos a dejar el tabaco } & \multirow{2}{*}{$O R$} & \multirow{2}{*}{ IC $95 \%$} & \multirow{2}{*}{$p$} \\
\hline & & $n$ & $\mathscr{F}(* *)$ & & & \\
\hline Total & 131 & 53 & 40.5 & & & \\
\hline \multicolumn{7}{|l|}{ SEXO } \\
\hline Masculino & 43 & 23 & 53.4 & & & \\
\hline Femenino & 88 & 30 & 34.1 & 2.2 & $1.1-4.7$ & .035 \\
\hline \multicolumn{7}{|l|}{ GRUPO ETARIO } \\
\hline$<31$ años & 13 & 8 & 61.5 & 1.0 & & \\
\hline $31-40$ años & 65 & 26 & 40.0 & 0.4 & $0.1-1.4$ & .160 \\
\hline $41-50$ años & 39 & 16 & 41.0 & 0.4 & $0.1-1.6$ & .205 \\
\hline$>50$ anos & 14 & 3 & 21.4 & 0.2 & $0.03-0.9$ & .041 \\
\hline \multicolumn{7}{|l|}{ ESTAMENTO } \\
\hline Cocina/lavandería & 5 & 0 & - & - & & \\
\hline Aux. Enfemería & 17 & 5 & 29.4 & 1.0 & & \\
\hline ATS/DUE & 50 & 17 & 34.0 & 1.2 & $0.4-4.1$ & .728 \\
\hline Facultativos & 22 & 9 & 40.9 & 1.7 & $0.4-6.4$ & .460 \\
\hline Administrativos & 19 & 10 & 52.6 & 2.7 & $0.7-10.6$ & .163 \\
\hline Celadores & 10 & 6 & 60.0 & 3.6 & $0.7-18.6$ & .126 \\
\hline Residentes & 4 & 3 & 75.0 & 7.2 & $0.6-87.0$ & .121 \\
\hline Mantenimiento & 4 & 3 & 75.0 & 7.2 & $0.6-87.0$ & .121 \\
\hline \multicolumn{7}{|c|}{ REGRESIÓN LOGÍSTICA MÚLTIPLE } \\
\hline & $O R$ & & & & $p$ & \\
\hline \multicolumn{7}{|l|}{ SEXO } \\
\hline Femenino & 1 & & & & & \\
\hline Masculino & 1.7 & & & & .362 & \\
\hline \multicolumn{7}{|l|}{ EDAD } \\
\hline$<31$ & 1 & & & & & \\
\hline$>50$ & 0.2 & & & & .094 & \\
\hline
\end{tabular}

(*) \% sobre los que fuman actualmente. 
dejar el hábito tabáquico El total está próximo a la mitad, siendo mayor la proporción de hombres, diferencia que sí resulta estadísticamente significativa. Entre los grupos de edad y tomando como referencia los más jóvenes, son estos los que muestran el mayor porcentaje de deseo de dejar de fumar. Sin embargo, en los mayores de 50 años se evidencia una OR menor, cstadísticamente significativa. Por estamentos ordenados de menor a mayor $y$ tomando como referencia el de menor porcentaje de deseo de dejar de fumar (auxiliares de enfermería) no hay diferencias estadísticamente significativas. Al valorar la asociación entre categorías de los que desean y no desean dejar el hábito, ajustado por todos los demás desaparece la significación estadística por sexo y edad.

En la tabla 5 se describen los trabajadores que declaran fumar en el hospital con expre- sión de los lugares más frecuentes, siendo éstos las salas de estar, despachos y cafetería. Sólo se ha podido constatar que dos de los 360 encuestados (un facultativo y un ATS/DUE) fuman en presencia de los pacientes.

Los consumidores de cigarrillos constituyen la inmensa mayoría frente a los que fuman otras labores (puros, pipas). En cuanto a cantidades diarias, se puede apreciar que el grupo mayoritario corresponde a la categoría intermedia (10-20 cigarrillos/día), mientras que los fumadores ocasionales y los grandes fumadores suponen el 8.7 y el $11.9 \%$ respectivamente (figura 1). En la distribución por sexos, grupos de edad y estamentos no se han detectado diferencias con respecto a la muestra total.

Al preguntar a los exfumadores sobre su consumo previo, se observa un patrón simi-

Tabla 5

Distribución de trabajadores que fuman en el hospital y lugares en los que fuman

\begin{tabular}{|c|c|c|c|c|c|c|c|c|}
\hline & \multicolumn{2}{|c|}{ Fuman Hospital } & \multicolumn{2}{|c|}{ Salas estar Despachos } & \multicolumn{2}{|c|}{ Delante enfermo } & \multicolumn{2}{|c|}{ Cafetería } \\
\hline & $n$ & $\% *$ & $n$ & $\% * *$ & $n$ & $\% * *$ & $n$ & $q * *$ \\
\hline Total & 111 & 84.7 & 89 & 80.2 & 2 & 1.8 & 62 & 55.9 \\
\hline \multicolumn{9}{|l|}{ SEXO } \\
\hline Masculino & 35 & 81.4 & 21 & 60.0 & 1 & 2.9 & 23 & 65.7 \\
\hline Femenino & 76 & 86.4 & 68 & 89.5 & 1 & 1.3 & 39 & 51.3 \\
\hline \multicolumn{9}{|l|}{ GRUPO ETARIO } \\
\hline$<31$ años & 11 & 84.6 & 8 & 72.7 & 0 & - & 8 & 72.7 \\
\hline 31-40 años & 54 & 83.1 & 42 & 77.8 & 1 & 1.9 & 35 & 64.8 \\
\hline 41-50 años & 34 & 87.2 & 29 & 85.3 & 0 & - & 14 & 41.2 \\
\hline$>50$ años & 12 & 85.7 & 10 & 83.3 & 1 & 8.3 & 5 & 41.7 \\
\hline \multicolumn{9}{|l|}{ ESTAMENTO } \\
\hline Facultativos & 17 & 77.3 & 14 & 82.4 & 1 & 5.9 & 13 & 76.5 \\
\hline ATS/DUE & 45 & 90.0 & 42 & 93.3 & 1 & 2.2 & 19 & 42.2 \\
\hline Aux. Enfermeria & 14 & 82.3 & 14 & 100 & 0 & - & 5 & 35.7 \\
\hline Celadores & 9 & 90.0 & 5 & 55.6 & 0 & - & 4 & 44.4 \\
\hline Cocina/Lavandería & 5 & 100 & 5 & 100 & 0 & - & 1 & 20.0 \\
\hline Mantenimiento & 3 & 75.0 & 0 & - & 0 & - & 3 & 100 \\
\hline Administrativos & 16 & 84.2 & 7 & 43.8 & 0 & - & 15 & 93.8 \\
\hline Residentes & 2 & 50.0 & 2 & 100 & 0 & - & 2 & 100 \\
\hline
\end{tabular}

* Porcentaje sobre el total de fumadores.

** Porcentaje sobre fumadores en el hospital. 
Figura 1

Cantidad de cigarrillos consumidos ( $\%$ de fumadores y ex-fumadores)

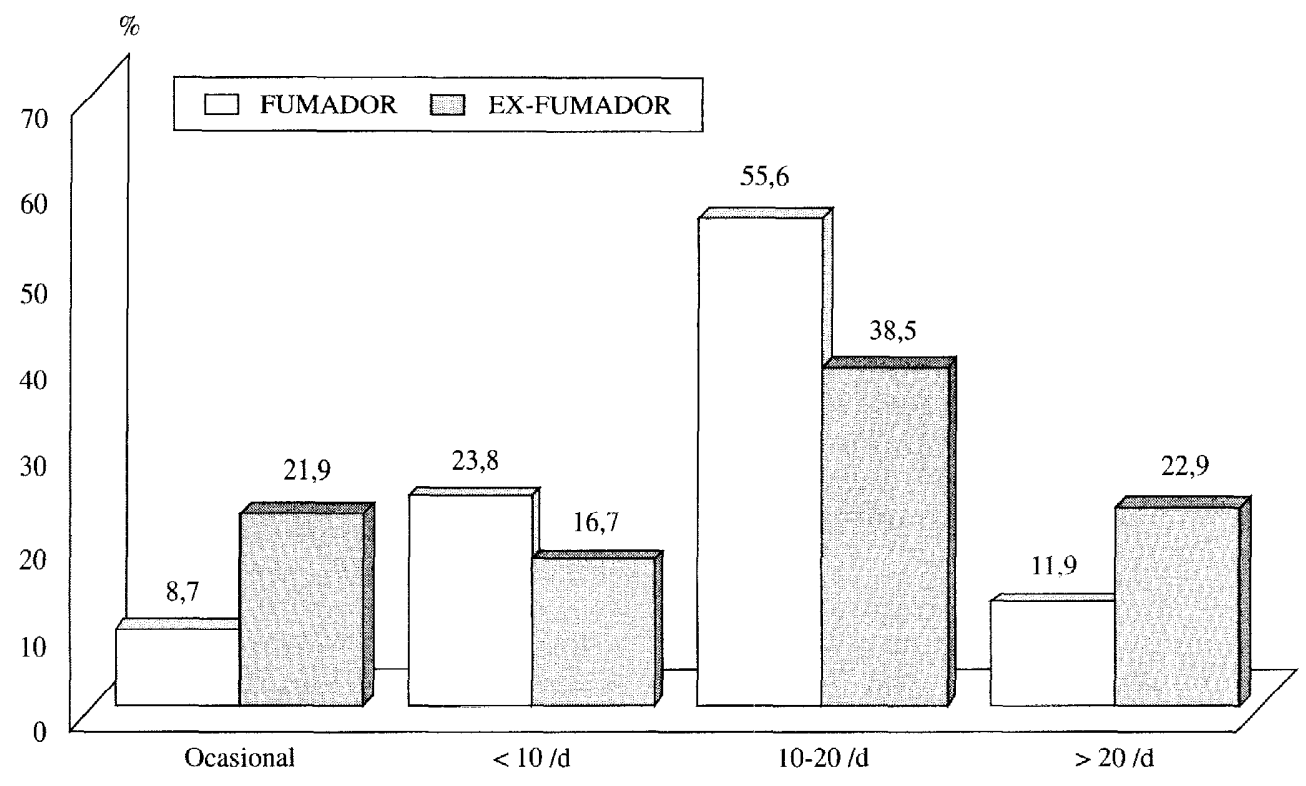

lar, pero llama la atención que los ocasionales y grandes fumadores suponen respectivamente el $21.9 \%$ y el $22.9 \%$, porcentajes muy superiores a los observados en los que siguen con el hábito de fumar.

Al solicitar de los fumadores que comentasen si habían tenido algún problema médico que pudiera estar relacionado con el tabaco, sólo $8(6.1 \%)$ contestan afirmativamente, de los cuales 4 señalan problemas de irritación de las vías altas del aparato respiratorio, 2 de ellos problemas vasculares, 1 neumonía y 1 problemas digestivos.

En cuanto al tiempo de consumo (figura 2), el conjunto de los que declaran llevar más de 15 años fumando es mayoritario, excepto, en el grupo de edad más joven y en médicos residentes. El tiempo que los exfumadores llevan sin fumar es muy variable, pero en su conjunto, se evidencia un mayor porcentaje de cesación del hábito durante los últimos cinco años (figura 3).

\section{DISCUSIÓN}

Consideramos la muestra de este estudio representativa de todos los estamentos del hospital, pero no extrapolable a los trabajadores de otros centros, ni a la población general. No obstante, en la muestra analizada, el porcentaje global de fumadores actuales $(36.4 \%$ ) (tabla 2 ) coincide con la Encuesta Nacional de Salud de 1993 de la población española ${ }^{9}$, aunque difiere en cuanto a sexos, siendo mayor para hombres $(48 \%)$ y menor para mujeres $(25 \%)$ que en la nuestra $(36.8 \%$ y $36.2 \%$ respectivamente).

También en el estudio epidemiológico sobre factores de riesgo cardiovasculares llevada a cabo en una muestra representativa de la población española en $1989^{10}$, se encuentra un porcentaje prácticamente igual en hombres (49.4\%) que en la Encuesta Nacional de Salud, pero menor en mujeres (16.7\%). 


\section{Figura 2}

Tiempo que llevan fumando

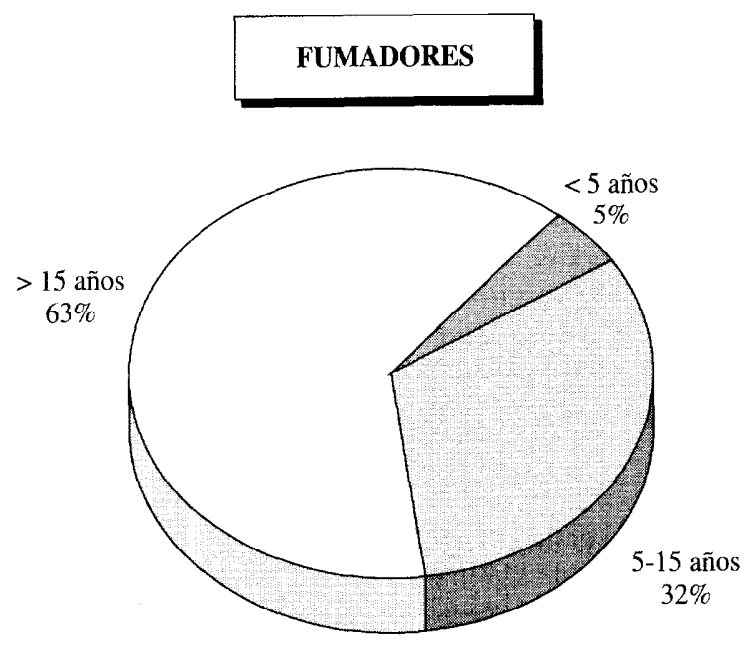

Figura 3

Tiempo de cesación del tabaquismo

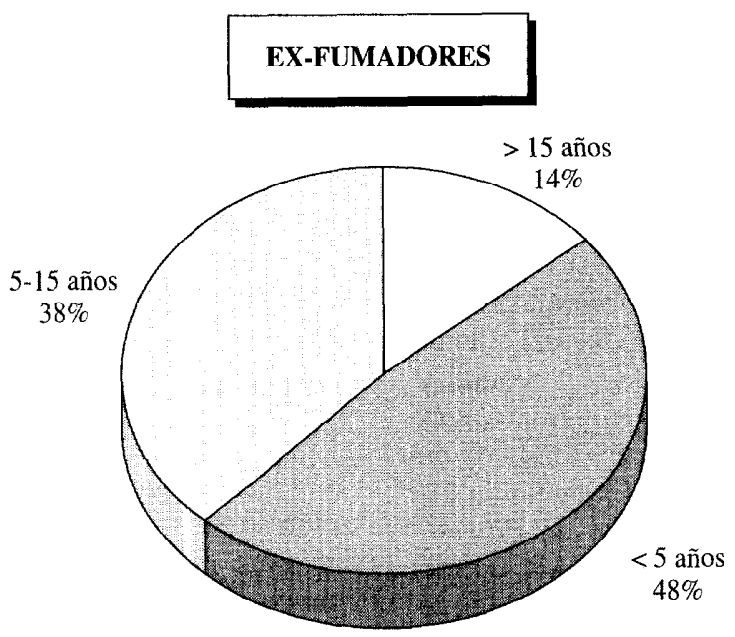


En la encuesta del Ministerio de Sanidad de $1978^{11}$ sobre el hábito de fumar de la población española, se obtuvo un $53.9 \%$ de fumadores en varones y $16.47 \%$ en mujeres. Sin embargo en mujeres entre 16 a 24 años la prevalencia se elevó al $48 \%$. Estos porcentajes indican una tendencia a una disminución global paulatina del hábito de fumar, la cual parece «resistirse» a disminuir más por la incorporación de las mujeres jóvenes $^{12}$ donde, según otros estudios, parece aumentar este porcentaje ${ }^{13,14}$. Esto ocurre en el presente trabajo en el que hay un elevado porcentaje de fumadores en los estamentos ATS/DUE y administrativos, donde las mujeres jóvenes son mayoría.

En otros países, se aprecia también una tendencia a la disminución global ${ }^{15}$ del hábito tabáquico, con un aumento en mujeres jóvenes ${ }^{16.17}$. Se constata que la prevalencia en la población adulta varía entre el 30 al $50 \%$ con predominio de varones sobre mujeres. Así se ha valorado en encuestas realizadas en Inglaterra ${ }^{18}$, Dinamarca ${ }^{19}$, USA $^{20}$ y Australia ${ }^{21}$.

En cuanto a la relación al hábito de fumar y trabajadores sanitarios, la cohorte de médicos británicos con los que fueron iniciados los estudios de Doll y Hill, citados anteriormente ${ }^{1-3}$, han continuado en el tiempo y se ha valorado la mortalidad en relación con el tabaco a los 10 años ${ }^{22}$, a $\operatorname{los} 20^{2.3}$ y a $\operatorname{los} 40^{24}$.

Fréour et $\mathrm{al}^{25}$, en una encuesta realizada a médicos franceses, observaron que la prevalencia del tabaquismo (43\%) era menor que la que había en la población general y también menor en médicos jóvenes, quizás por una mejor educación antitabáquica en las facultades de medicina. En cuanto a los médicos españoles, el Comité de Prevención de Tabaquismo de la SEPAR estimó que el porcentaje de fumadores disminuyó de un $49.2 \%$ en $1985^{26}$, situado por encima de la media de la población general $(39 \%)$ al $37 \%$ en $1990^{27}$ por debajo de la media $(40 \%)$ y muy similar a nuestros datos
(35.5\%). En Barcelona ${ }^{28}$, el porcentaje de disminución observado en médicos varones entre 1981 y 1985 quedó compensado por el incremento en las médicas.

Por lo que respecta al colectivo ATS/DUE, los datos de nuestra muestra son muy similares a los encontrados en el estudio multicéntrico de 1990 en hospitales españoles y portugueses ${ }^{27}$ ( $48.5 \%$ vs $47.6 \%$ ), aunque difieren en el grupo de auxiliares de enfermería, ya que en nuestro estudio el porcentaje es menor ( $41.5 \%$ vs $25 \%$ ).

En una encuesta reciente sobre tabaquismo en trabajadores sanitarios en un área de Atención Primaria de salud en Madrid ${ }^{29}$, se obtuvieron unos datos muy similares a los nuestros tanto en fumadores actuales (diarios y ocasionales), sexo y edad, así como la proporción de cesación.

En estudiantes de medicina, también se detecta un descenso, sobre todo en el período clínico $^{30}$ y una menor prevalencia en estudiantes de carreras sanitarias con respecto a otro tipo de estudios ${ }^{31}$. En Santiago de Compostela ${ }^{32}$, llegan a estos mismos resultados después de un seguimiento de 11 años ${ }^{33}$.

La encuesta detecta un importante número de personas que han dejado de fumar (13.6 al $65 \%$ ) y sobre todo los que han cesado en los últimos 5 años (figura 3). Por otra parte, si tenemos en cuenta el porcentaje de fumadores que se declaran dispuestos a dejar de fumar $(40.5 \%$ del total) (tabla 4$)$, anima a proseguir con acciones conducentes para ayudar a la cesación del hábito. Estas actividades se basan en tres estrategias:

1. Educación para la salud con información sobre los efectos perjudiciales del tabaco para el fumador y los demás (fumador pasivo ${ }^{34}$.

2. Consejo médico antitabáquico y utilización de una serie de técnicas de profilaxis a este respecto ${ }^{35,36}$. 
3. Medidas legislativas. Obligar al cumplimiento del Real Decreto número 192/88 ${ }^{37}$ sobre limitación en la venta y uso del tabaco, el cual establece expresamente la prohibición de fumar en centros públicos y cita especialmente a hospitales.

De acuerdo con las estrategias de la OMS de Salud para todos en el año $2000^{38}$ y en el ámbito de acciones de promoción de la salud, proponemos el proyecto de declarar, a partir de ese año, al hospital como hospital sin tabaco en el que nadie, absolutamente nadie, fume. Esto debe incluir a: trabajadores de todos los estamentos, pacientes ingresados y visitantes. El hospital sin tabaco tendría tres beneficios: mejora de la limpieza general, supresión de un foco de contaminación ambiental (humo del tabaco) y función ejemplarizante (educación para la salud).

\section{AGRADECIMIENTOS}

A D. Jesús Curiel, Gerente del Hospital en el momento de realizar este estudio, por las facilidades dadas y a todas las personas que con gran entusiasmo colaboraron en ella: miembros de la dirección de personal, grupo de prevención del uso del tabaco, servicio de medicina preventiva y salud pública, así como a los estudiantes de sexto curso de medicina de la unidad docente de Vitoria (curso 1994-95).

\section{BIBLIOGRAFÍA}

1. Doll R. Hill AB. Smoking and carcinoma of the lung. Preliminary report. BMJ 1950; II: 739-48.

2. Doll R. Hill AB. The mortality of doctors in relation to their smoking habits. A preliminary report. BMJ 1954: I: 451-5.

3. Doll R. Hill AB. Lung cancer and other causes of death in relation to smoking. A second report on the mortality of British doctors. BMJ 1956; II: 1071-6.

4. Wiley JA. Camacho TC. Life style and Future Health: Evidence froom the Alameda County Study. Prev Med 1980; 9: 1-21.
5. US Department of health and Welfare. Sinoking and Health. A report of the surgeon general Washington: Department of health and Welfare; 1979. Pub 79-50066.

6. Dawbert TR. The Framinghan study. Cambridge. Masachusets: Harvard University Press; 1980.

7. Sierra Bravo R. Tamaño de las muestras. Técnicas de Investigación Social. Madrid: Paraninfo; 1989. p. 226-38.

8. Silva LC. Muestreo para la investigación en Ciencias de la Salud. Madrid: Díaz de Santos; 1993.

9. Arévalo R, Banegas JR, Biglino L, et al. Encuesta Nacional de Salud 1993. Rev San Hig Púb 1994: 68: 127-78.

10. Banegas JR, Villar F, Pérez de Andres C, et al. Estudio epidemiológico de los factores de riesgo cardiovasculares en la población española de 35 a 64 años. Rev San Hig Púb 1993; 67: 419-45.

11. Ministerio de Sanidad y Seguridad Social. Encuesta Nacional sobre consumo de tabaco. Bol Epidemiol Semanal 1981; 1502: 289-91.

12. De Onis M, Villar J. Tobacco use in Spanish women. World Health Statist Q 1991; 44: 80-8.

13. Gestal JJ, Montes A, Barros JM, Smyth E. Tabaco y Patología La Coruña: Real Academia de Medicina y Cirugía de Galicia; 1988.

14. Nebot M, Borrell C, Ballestín M, Villalbí JR. Prevalencia y características asociadas al consumo de tabaco en población general en Barcelona entre 1983 y 1992. Rev Clin Esp 1996; 196: 359-64.

15. Pierce JP. International comparisons of trends in cigarette smoking prevalence. Am J Public Health 1989; 79: 152-7.

16. Ernster VL. Trends in smoking, cancer risk and cigarette promotion. Cancer 1988; 62: 1702-12.

17. Anónimo. Smoking and health: a 25-year perspective (editorial). Am J Public Health, 1989: 79 (2): 141-3.

18. Russell MA, Wilson C, Taylor C, Baker CD. Smoking prevalence among general practitioner's patients. Br J Addict 1980; 75: 367-73.

19. Roed-Petersen BJ. Smoking habits in East Denmark at october 1979. Scand J Soc Med 1984; 12 : $39-47$.

20. Gottlieb NH, Green LW. Life events social network life-style and health an analysis. Nueva York: John Wiles \& Jons Inc; 1984. 
21. Hill DJ, Gray NJ. Patterns of tobacco smoking in Australia. Med J Aust 1982; 1: 23-5.

22. Doll $\mathrm{R}$, Hill $\mathrm{AB}$. Mortality in relation to smoking: years'observations on British doctors. BMJ 1964; i: $1399-414,1460-7$

23. Doll $\mathrm{R}$, Peto $\mathrm{R}$. Mortaly in relation to smoking: 20 years' observation on male British doctors. BMJ 1976; ii: 1525-36.

24. Doll R, Peto R, Wheatley K, Gray R, Sutherland I. Mortality in relation to smoking: 40 years' observations on male British doctors. BMJ 1994; 309: 901-11.

25. Fréour P, Tessier JF, Gachie JP et al. Le tabaquisme chez les praticiens girondins. Bordeaux Méd $1980 ; 13$ (7): 253-60.

26. Esteras A. Prevalencia del hábito tabáquico entre médicos españoles. Arch Bronconeum 1986; 22 (supl 1): 57.

27. Sánchez Agudo L, De Padua J, Costa S, et al. Smoking Prevalence in Spanish and Portuguese hospitals. Results of a multicenter study of a programme for the implementation of a smoking control policy. 8th World Conference on Tobacco or Health. Buenos Aires; 30 Marzo-3 Abril 1992.

28. Salleras L, Pardell H, Canela J, Vaqué J, Plans P. Actitudes y hábitos tabáquicos de los médicos de Cataluña (España). Med Clin (Barc) 1987; 89: 6-9.

29. Ortiz H, Palancar JL, Cañamares V, Molina W, González C, Navarro A. El tabaquismo en los trabajadores de Atención Primaria del Área Sanitaria n. ${ }^{\circ} 4$ del Insalud de Madrid: prevalencia de consumo y actitudes. Rev Esp Salud Pública 1996; 70: 295-305
30. Knopf A. Changes in the smoking behaviour knowledge and opinion of medical students $1972-$ 1981. Soc Sci Med 1982: 16: 2137-43.

31. Ashley MJ. Smoking habits knowledge and attitudes of final year university students in the health profesions. Pre Med Toronto 1981; 10: 645-54.

32. Gestal JJ, Montes A. Smoking habits of final years Galician medical students. Rev Epidemiol Santé Publique 1987; 35: 386-92.

33. Montes A, Gestal IJ. Fvolución del háhito tabáquico en estudiantes de medicina: 11 años de estudio. VIII Congreso Nacional de la Sociedad Española de Higiene y Medicina Preventiva Hospitalaria. Vitoria; 20-22 Septiembre, 1995.

34. Salleras L. Lucha contra el hábito de fumar. En: Educación Sanitaria. Principios, métodos y aplicaciones. Madrid: Díaz de Santos: 1990. p. $237-$ 268 .

35. Gestal JJ, Montes A, Barros JM, Smyth E. Profilaxis del tabaquismo. En: Tabaco y Patología 1988; 140-172. Fundación Pedro Barrié de la Maza. La Coruña: Real Academia de Medicina y Cirugía; 1978.

36. Salleras L, Pardel H, Saltó E, et al. Consejo antitabaco. Med Clin (Barc) 1994; 102 (supl 1): 109 117.

37. Real Decreto 4 de marzo n. 192/88 sobre limitaciones en la venta y uso del tabaco para protección de la salud de la población. BOE núm. 59 del $9 / 3 / 88$.

38. WHO. Global strategy for health for all by the year 2000. Ginebra: WHO: 1981 